\title{
INFLUÊNCIA DA CONCENTRAÇÃO DO SAL E DA TEMPERATURA NA DETERMINAÇÃO DOS COEFICIENTES DE ATIVIDADE EM DILUIÇÃO INFINITA DE SISTEMAS ETANOL/ÁGUA/SAL POR SPME-GC/FID
}

\author{
B. R. dos SANTOS ${ }^{1}$, A.M. ELIAS ${ }^{1}$ e G.L.V. COELHO ${ }^{1}$ \\ ${ }^{1}$ Universidade Federal Rural do Rio de Janeiro, Departamento de Engenharia Química \\ E-mail para contato: coelho@ufrrj.br
}

\begin{abstract}
RESUMO - Neste trabalho, a micro extração em fase sólida (SPME) aliada a cromatografia gasosa foi utilizada para avaliar a influência de diferentes concentrações de sais inorgânicos no coeficiente de atividade em diluição infinita em sistemas etanol-água-sal nas temperaturas de 323,15, 333,15, 343,15 e 353,15 K. A técnica utilizada é rápida, de baixo custo e de fácil implementação, que aliada a um novo equacionamento termodinâmico, permitiu determinar o coeficiente de atividade em diluição infinita avaliando a concentração de sal na solução. Os sais estudados foram Cloreto de Potássio $(\mathrm{KCl})$ e Cloreto de Cálcio $\left(\mathrm{CaCl}_{2}\right)$, em concentrações de 1,125, 1,800 e 2,571 mols/kg de água. $\mathrm{O} \mathrm{CaCl}_{2}$ apresentou a melhor influência na separação do álcool estudado.
\end{abstract}

\section{INTRODUÇÃO}

Um soluto está infinitamente diluído quando as moléculas deste soluto estão completamente envolvidas por moléculas de solvente. Neste caso, a fração molar do soluto em solução tende a zero e a fração molar do solvente tende a um. Nesta conformação a não idealidade deste soluto em solução é máxima, sendo representada pelo coeficiente de atividade em diluição infinita $\left(\gamma^{\infty}\right)$. Tais coeficientes são de grande importância, visto que uma vez determinados é possível predizer o equilíbrio entre dois componentes determinando-se os parâmetros de modelos matemáticos que descrevem as variações das energias de excessos e atividade dos componentes dessas misturas. Com isso, equipamentos que têm como fundamentação básica o equilíbrio entre fases podem ser projetados com maior precisão, obtendo-se compostos mais puros. Os coeficientes de atividade em diluição infinita podem ainda ser utilizados para a seleção de solventes capazes de remover de uma solução um dado componente específico, definindo um parâmetro denominado seletividade (Elias et al, 2014).

O efeito do sal se dá com o aumento da dissociação de eletrólitos no sistema. Essas forças causam a formação de complexos com a fase líquida. Quando um sal é adicionado, as propriedades da mistura podem ser alteradas, esse fenômeno é conhecido como salting in, que reduz a volatilidade do componente que forma os complexos, e salting out, que aumenta a volatilidade do outro componente. O efeito do sal no equilíbrio líquido-vapor é importante para diferentes processos de separação industrial, como a destilação extrativa. A adição de sal também pode mudar a solubilidade relativa em extração líquido-líquido, e em sistemas de 
mistura de solventes provoca um processo de solvatação onde a molécula central, que nesse estudo é a água, envolve os íons do sal, liberando o soluto (Banat et al., 2003).

Muitas técnicas vêm sendo desenvolvidas ao longo dos anos para a determinação dos coeficientes de atividade em diluição infinita para misturas líquidas, sendo algumas mais complexas e custosas, como o GLC (Gas-Liquid Chromatography), e absorção gasosa com gás inerte não solúvel (Gas-Stripping) (Furtado e Coelho, 2010).

Neste trabalho a micro extração em fase sólida SPME será utilizada aliada a cromatografia a gás GC-FID para realizar uma extração no headspace HS, a fím de se determinar o coeficiente de atividade em diluição infinita. Uma técnica que leva vantagens sobre as outras, por ser rápida, acurada, confiável e de fácil aplicação (Valente e Augusto, 2000).

\section{PARTE EXPERIMENTAL}

Os reagentes de grau analítico utilizados foram obtidos da Vetec Química Fina Ltda sendo: etanol e orto-xileno, com pureza superior a $99,4 \%$, verificado por cromatografia gasosa; os sais cloreto de potássio e cloreto de cálcio anidro com pureza acima de $99 \%$ Os sais antes da sua utilização ficaram na mufla a $398,15 \mathrm{~K}$ por pelo menos $5 \mathrm{~h}$, para que a umidade fosse reduzida. A fibra de PDMS (polidimetilsiloxano) de espessura $100 \mu \mathrm{m}$ foi obtida da Supelco. O cromatógrafo a gás utilizado no experimento foi um GC-2010 Shimadzu equipado com detector de ionização em chama (FID = Flame Ionization Detector), uma coluna capilar HP-Innowax (polietileno glicol reticulado; crosslinked PEG) de dimensões 60 $\mathrm{m} \times 0,32 \mathrm{~mm}$ x 0,25 $\mu \mathrm{m}$, um liner da SGE Analytical Science Pty Ltd, de diâmetro interno $0,75 \mathrm{~mm}$ (próprio para SPME) e hélio ultrapuro $(99,999 \%)$ como gás de arraste. Frascos âmbar de $40 \mathrm{~mL}$ com septo de PTFE/silicone serviram para o confinamento e estudo das misturas líquidas. Foram utilizadas seringas cromatográficas com volumes de 10, 100, 500 e $1000 \mu$ l obtidas da HAMILTON.

A curva de calibração para o etanol foi construída injetando-se volumes precisos de 1 $\mu \mathrm{L}$ de solução com concentrações variadas dos componentes em orto-xileno, no injetor do cromatógrafo a gás. A pressão no injetor foi mantida constante a $123,60 \mathrm{kPa}$ em todo o período de análise, utilizando o modo splitless por 2 minutos e em seguida uma razão de split igual a $1: 8$, a temperatura no injetor e detector foram mantidas a $250^{\circ} \mathrm{C}$. A temperatura inicial da coluna foi $50^{\circ} \mathrm{C}$, mantida por 5 minutos, elevando-se a $80^{\circ} \mathrm{C}$ a uma taxa de aquecimento de $10^{\circ} \mathrm{C} \cdot \mathrm{min}^{-1}$, mantendo-se nesta temperatura por mais 1 minuto.

A determinação do coeficiente de partição fibra-gás $\left(\mathrm{K}_{\mathrm{fg}}\right)$ se deu através da injeção de 1 $\mu \mathrm{L}$ de etanol em frascos âmbar de $40 \mathrm{~mL}$. A temperatura do sistema foi controlada por um banho termostático (LAUDA, modelo RM 6B). O tempo de extração da fibra no headspace foi determinado, onde a massa extraída não mais variasse com o tempo de exposição na menor temperatura. A determinação do $\mathrm{K}_{\mathrm{fg}}$ foi realizada para as 4 temperaturas estudadas nas mesmas condições cromatográficas da curva de calibração.

Foram preparadas soluções de $8,39,13,42,19,17 \mathrm{~g}$ para $0,1 \mathrm{~kg}$ de água para o $\mathrm{KCl}$ e de $12,49,19,98$ e $28,54 \mathrm{~g}$ para $0,1 \mathrm{~kg}$ de água para o $\mathrm{CaCl}_{2}$, sendo estas soluções com os respectivos números de mols/kg de água de 1,125, 1,800 e 2,571 para os dois sais. 
A extração do analito no headspace através da SPME ocorreu em frascos âmbar de 40 $\mathrm{mL}$, onde $10 \mathrm{~mL}$ de solução em cada concentração molal estudada foi confinado juntamente com $1,5 \mu \mathrm{L}$ de etanol. A mistura permaneceu sob agitação magnética a temperatura constante por 45 minutos e por mais 45 minutos em repouso. A extração com a fibra ocorreu no tempo de 20 min para garantir a absorção do soluto pela fibra.

A temperatura do sistema foi controlada por um controlador do tipo PID com uma termoresistência do tipo PT-100 (erro de $\pm 0,1 \mathrm{~K}$ ). Após a extração, a fibra de PDMS foi exposta no injetor do cromatógrafo a gás para a quantificação de material extraído, sendo mantidas as mesmas condições da construção da curva de calibração.

Todas as análises foram realizadas em quadruplicatas para uma melhor confiabilidade dos resultados obtidos.

\section{RESULTADOS E DISCUSSÕES}

A construção da curva de calibração apresentou boas correlações, tendo como coeficiente angular de 9723 e coeficiente de determinação $\left(\mathrm{R}^{2}\right)$ 0,9999.

Os coeficiente de partição fibra-gás $\left(\mathrm{K}_{\mathrm{fg}}\right)$ foram determinados para os solutos estudados através da Equação 1, onde $\mathrm{V}_{\mathrm{f}}$ é o volume da fibra, $\mathrm{V}_{\mathrm{g}}$ o volume do headspace do frasco, $\mathrm{n}_{1}{ }^{\mathrm{f}}$ número de mols do soluto absorvido pela fibra e $\mathrm{n}_{1}{ }^{\mathrm{g}}$ número de mols do soluto na fase gasosa do sistema.

$$
K_{f g}=\frac{n_{1}^{f}}{V_{f}} \frac{V_{g}}{n_{1}^{g}}
$$

Para verificar a consistência dos dados experimentais, foi realizada uma linearização dos dados, verificando-se a confiabilidade dos dados, que apresentou um coeficiente de determinação $\left(\mathrm{R}^{2}\right)$ de 0,9993 como mostra a Figura 1.

Figura 1 - Linearização do coeficiente de partição fibra-gás $\left(\mathrm{K}_{\mathrm{fg}}\right)$.



A partir dos dados de coeficiente de partição fibra-gás, pode-se obter através da Equação 2 o coeficiente de partição líquido-gás $\left(\mathrm{K}_{\mathrm{Lg}}\right)$ para o soluto em cada temperatura e concentração de sal estudado, onde $\mathrm{n}_{\mathrm{o}}{ }^{\mathrm{L}}$ é o número de mols iniciais do soluto em solução e $\mathrm{V}_{\mathrm{L}}$ o volume de solução. 


$$
K_{L g}=\left[K_{f g} V_{f}\left(\frac{n_{o}^{L}}{n_{1}^{f}}-1\right)-V_{g}\right] \frac{1}{V_{L}}
$$

Para o correto cálculo do coeficiente de atividade em diluição infinita $\left(\gamma_{i}^{\infty}\right)$ precisamos de certas variáveis como a densidade do solvente $\left(\rho^{\mathrm{L}}{ }_{2}\right)$ e a pressão de saturação do soluto $\left(\mathrm{P}_{1}{ }^{\text {sat}}\right)$. A pressão de vapor do soluto em cada temperatura foi calculada a partir da equação de Wagner, com os parâmetros encontrados na literatura. $\mathrm{O}$ segundo coeficiente de virial $\left(\mathrm{B}_{11}\right)$ foi determinado pelo método de Tsonopoulos e o volume molar do soluto $\left(\mathrm{v}_{1}{ }^{0}\right)$ foi determinado pela equação empírica de Rackett. As equações e os parâmetros estão disponíveis na literatura (Reid et al., 1987).

Através da Equação 3 e com os parâmetros determinados, pode-se determinar o coeficiente de atividade em diluição infinita dos compostos, onde $\mathrm{T}$ é a temperatura, $\mathrm{R}$ a constante dos gases, $\mathrm{n}_{\text {sal }}$ é o número de mols em solução, $\mathrm{n}_{2}{ }^{\mathrm{L}}$ é o número de mols da água em solução e $\mathrm{n}_{1}{ }^{0}$ é o número de mols iniciais do soluto adicionado. Os valores encontrados estão demonstrados na Tabela 1 para o $\mathrm{KCl}$ e $\mathrm{CaCl}_{2}$.

$$
\gamma_{1}=\frac{1}{P_{1}^{s a t}} \frac{n_{1}^{f} R T}{K_{f g} V_{f}}\left(\frac{K_{f g} V_{f}\left(n_{s a l}+n_{2}^{L}+n_{1}^{0}-n_{1}^{f}\right)-n_{1}^{f} V_{g}}{K_{f g} V_{f}\left(n_{1}^{0}-n_{1}^{f}\right)-n_{1}^{f} V_{g}}\right)
$$

\begin{tabular}{|c|c|c|c|c|c|c|c|c|}
\hline \multirow{3}{*}{$\begin{array}{c}\text { Concentração } \\
\text { (mols/kg de } \\
\text { água) }\end{array}$} & \multicolumn{8}{|c|}{$\gamma_{i}^{\infty}$ médio } \\
\hline & \multicolumn{2}{|c|}{$\mathrm{T}(323,15 \mathrm{~K})$} & \multicolumn{2}{|c|}{$\mathrm{T}(333,15 \mathrm{~K})$} & \multicolumn{2}{|c|}{$\mathrm{T}(343,15 \mathrm{~K})$} & \multicolumn{2}{|c|}{$\mathrm{T}(353,15 \mathrm{~K})$} \\
\hline & $\mathrm{KCl}$ & $\mathrm{CaCl}_{2}$ & $\mathrm{KCl}$ & $\mathrm{CaCl}_{2}$ & $\mathrm{KCl}$ & $\mathrm{CaCl}_{2}$ & $\mathrm{KCl}$ & $\mathrm{CaCl}_{2}$ \\
\hline 1,125 & $7,87 \pm 0,11$ & $9,02 \pm 0,16$ & $7,52 \pm 0,08$ & $8,47 \pm 0,32$ & $7,19 \pm 0,15$ & $8,07 \pm 0,15$ & $6,92 \pm 0,08$ & $7,65 \pm 0,13$ \\
\hline 1,800 & $10,61 \pm 0,26$ & $11,89 \pm 0,37$ & $9,83 \pm 0,14$ & $10,86 \pm 0,25$ & $9,24 \pm 0,24$ & $10,05 \pm 0,29$ & $8,52 \pm 0,30$ & $9,52 \pm 0,10$ \\
\hline 2,571 & $13,83 \pm 0,25$ & $16,36 \pm 0,07$ & $13,05 \pm 0,08$ & $15,01 \pm 0,14$ & $11,92 \pm 0,12$ & $13,54 \pm 0,18$ & $11,26 \pm 0,08$ & $12,33 \pm 0,11$ \\
\hline
\end{tabular}

Tabela 1 - Coeficiente de atividade em diluição infinita com a adição do $\mathrm{KCl}$ e $\mathrm{CaCl}_{2}$

Os resultados obtidos foram comparados com os da literatura. Nota-se que com o aumento da temperatura, há uma diminuição do coeficiente de partição fibra-gás $\left(\mathrm{K}_{\mathrm{fg}}\right)$ o que também pode ser visto no coeficiente de atividade em diluição infinita $\left(\gamma_{i}^{\infty}\right)$. O $\gamma_{i}^{\infty}$ aumentou com o acréscimo de sal, mostrando o aumento do efeito salting out provocado pela interação íon-solvente, onde as partículas de sal dissolvido tendem a atrair preferencialmente a um tipo de molécula de solvente do que a de outro, onde geralmente esta atração ocorre para a molécula mais polar e com isso a fase vapor é enriquecida com o componente menos polar. $\mathrm{O}$ $\mathrm{KCl}$ apresentou o menor desvio de $28,14 \%$ e o maior de $251,87 \%$ se comparado com o $\gamma_{i}^{\infty}$ da literatura sem adição de sal. Já o $\mathrm{CaCl}_{2}$ apresentou o menor desvio de $43,92 \%$ e o maior de $285,42 \%$ se comparado também com o $\gamma_{i}^{\infty}$ da literatura sem adição de sal, como pode ser observado na Figura 2 e na Figura 3 (Banat et al., 2003).

Figura 2 - Comparação dos resultados experimentais do $\gamma_{\mathrm{i}}^{\infty}$ para o $\mathrm{KCl}$ em cada $\mathrm{T}$ e Concentração com os dados da literatura. 


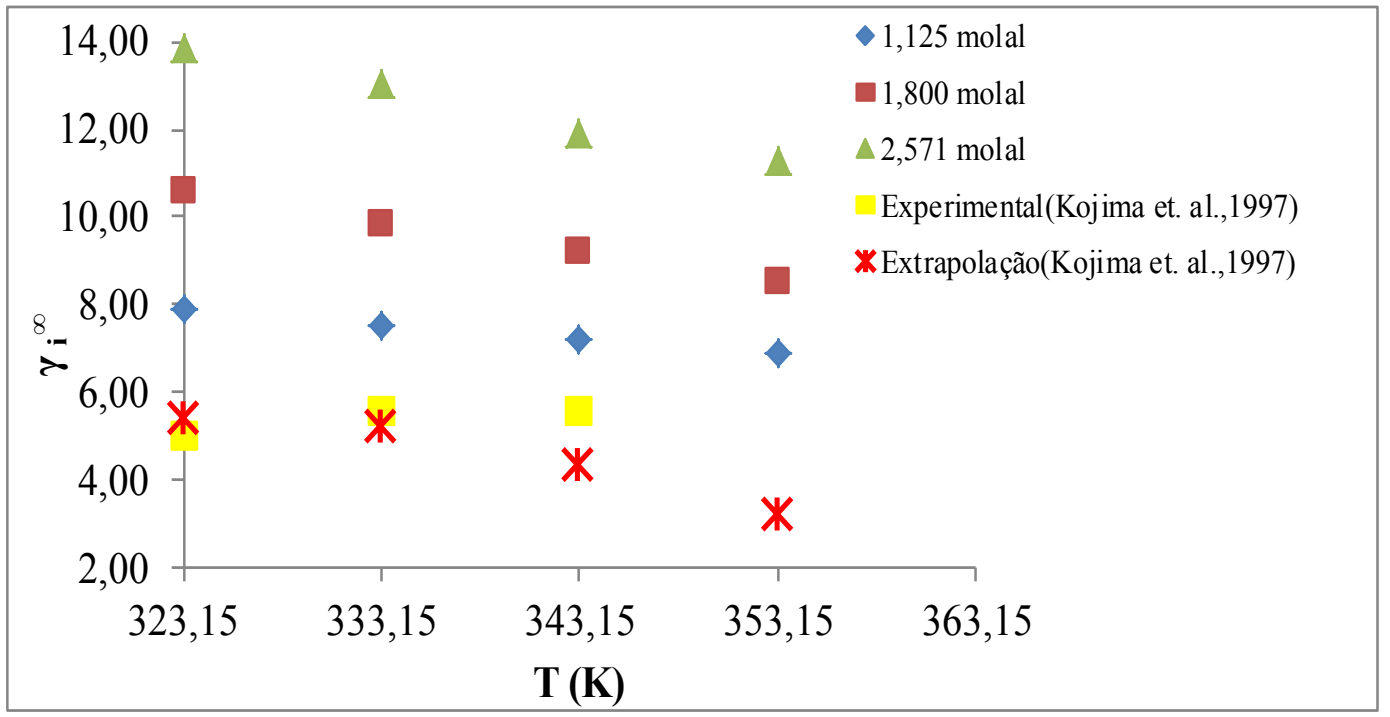

Figura 3 - Comparação dos resultados experimentais do $\gamma_{i}^{\infty}$ para o $\mathrm{CaCl}_{2}$ em cada $\mathrm{T}$ e Concentração com os dados da literatura.

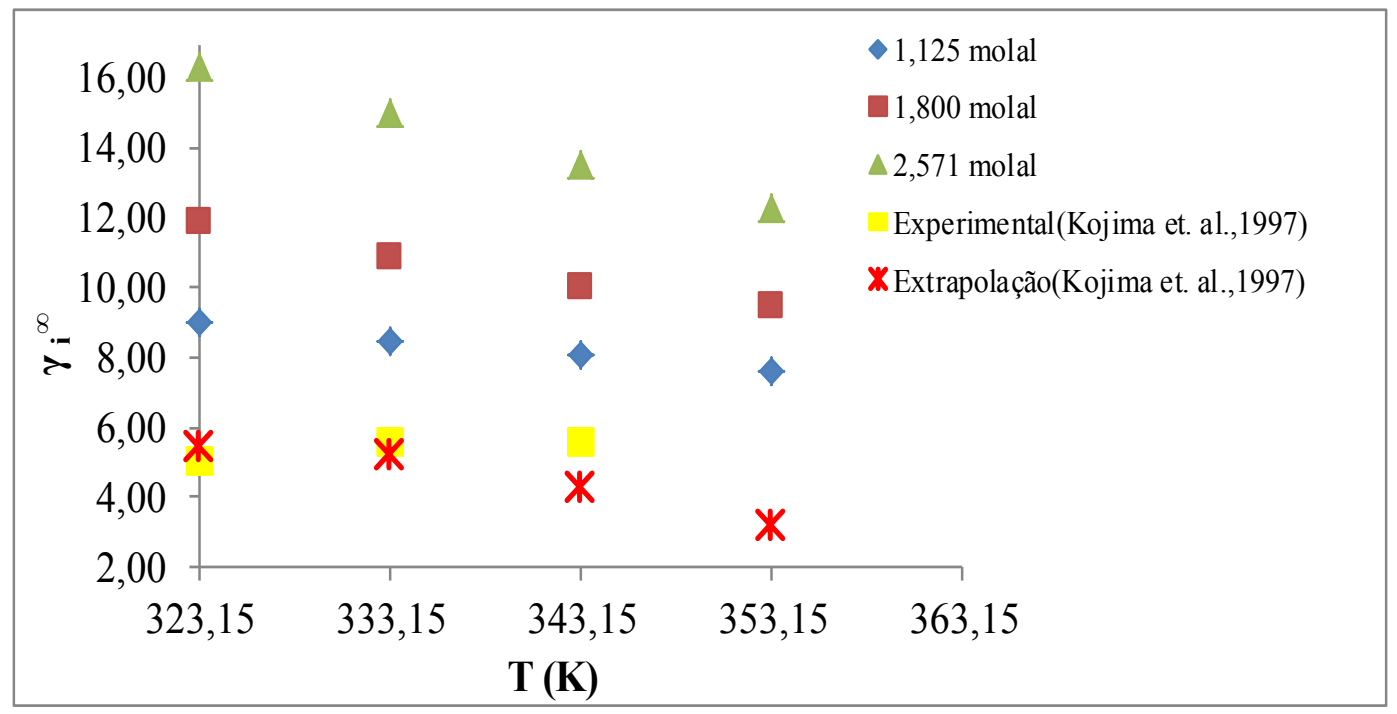

Com o aumento da força iônica, devido ao aumento do sal dissolvido, aumenta-se o longo alcance das interações eletrostáticas que são baseadas na teoria de Debye/Huckel. Logo, de acordo com a teoria eletrostática, a diminuição do raio iônico provoca um aumento do campo eletrostático, aumentando o efeito de salting out. Isto é evidenciado neste trabalho, onde o $\mathrm{KCl}$ e $\mathrm{CaCl}_{2}$, que se dissociam em dois e em três íons respectivamente, tendo o $\mathrm{K}^{+1} \mathrm{um}$ raio de $1,33 \AA$ e o $\mathrm{Ca}^{+2}$ um raio de $0,99 \AA$, comprovando que o efeito salting out é maior para o $\mathrm{CaCl}_{2}$ (Banat et al., 2003).

\section{CONCLUSÃO}

A SPME aliada ao GC-FID se mostrou como uma técnica viável para a determinação do coeficiente de atividade em diluição infinita $\left(\gamma_{i}^{\infty}\right)$ por ser rápida, confiável e de fácil 


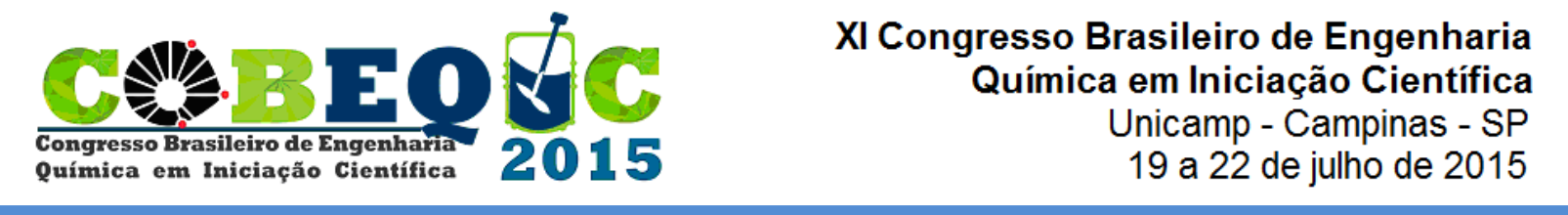

aplicação. Conclui-se que a adição de sal altera as condições termodinâmicas do sistema e que quanto menor o raio iônico maior será o campo eletrostático, aumentando o efeito salting out e consequentemente o $\gamma_{i}^{\infty}$.

Em todas as concentrações estudadas o $\mathrm{CaCl}_{2}$ foi que apresentou o maior percentual de distanciamento do coeficiente de atividade em diluição infinita sem sal da literatura, mostrando uma maior separação que o $\mathrm{KCl}$. Tendo o $\mathrm{KCl}$ o menor distanciamento de $28,14 \%$ para a concentração de $1,125 \mathrm{mols} / \mathrm{kg}$ de água na temperatura de $343,15 \mathrm{~K}$ e o maior percentual de separação de $251,87 \%$ na concentração de $2,571 \mathrm{mols} / \mathrm{kg}$ de água e temperatura de $353,15 \mathrm{~K}$. Já o $\mathrm{CaCl}_{2}$ teve o seu menor distanciamento da literatura de $43,92 \%$ e o maior de $285,42 \%$ nas mesmas condições do $\mathrm{KCl}$ na concentração de 1,125 e 2,571 mols $/ \mathrm{kg}$ de água respectivamente e nas temperaturas de 343,15 e $353,15 \mathrm{~K}$, respectivamente. Logo, o aumento do efeito salting out é evidenciado com o aumento da concentração do sal e da temperatura e com a diminuição do raio iônico.

\section{REFERÊNCIAS}

BANAT, F.; ASHEH, S. A.; SIMANDL, J.; Effect of trivalent, bivalent, and univalent cation inorganic salts on the isothermal vapor-liquid equilibria of propionic acid-water system, Chem. Eng. and Proc. v. 42, p. 759-766, 2003

ELIAS, A. M.; FURTADO, F. A; COELHO, G. L. V.; Determinação do coeficiente de atividade na diluição infinita em sistemas etanol-água-sal por microextração em fase sólida-gc-fid, Quim. Nova, v. 37, p. 1177-1181, 2014

FURTADO, F. A.; COELHO, G. L. V.; Determinação do coeficiente de atividade em diluição infinita de hidrocarbonetos em furfural a 298,15 k por SPME-GC/FID, Quim, Nova, v, 33, p, 1905-1909, 2010

KOJIMA, K.; ZHANG, S.; HIAKI, T.; Measuring methods of infinite dilution activity coefficients and a database for systems including water, Fluid Phase Equil., v. 131, p. $145-179,1997$

REID, R. C.; PRAUSNITZ, J. M.; POLING, B. E.; The Properties of Gases and Liquids, $4^{\mathrm{a}}$ Edição, New York, Mc Graw Hill, 1987

Os autores agradecem à FAPERJ pelo apoio financeiro. 